\title{
Laparoscopic Dismembered Repair in Two Patients with Retrocaval Ureter
}

\author{
Yuri Cavaleri ${ }^{1}$ Giuseppe Farullo ${ }^{1}$ Simona Gerocarni Nappo ${ }^{2}$ Paolo Caione ${ }^{3}$ \\ ${ }^{1}$ Department of Surgery, Urology UOSD, University of Rome Tor \\ Vergata, Roma, Lazio, Italy \\ 2 Department of Nephrology and Urology, Bambino Gesù Children's \\ Hospital, IRCCS, Rome, Italy \\ ${ }^{3}$ Division of Pediatric Urology, Bambino Gesù Children's Hospital, \\ IRCCS, Rome, Italy \\ Eur J Pediatr Surg Rep 2020;8:e32-e34. \\ Address for correspondence Paolo Caione, MD, Division of Pediatric \\ Urology, Bambino Gesù Children's Hospital, IRCCS, Piazza S. \\ Onofrio, 4 Rome 00165, Italy (e-mail: pcaione@yahoo.it).
}

\author{
Abstract \\ Keywords \\ - retrocaval ureter \\ - ureteroureterostomy \\ - laparoscopy
}

Retrocaval ureter (RCU) or circumcaval ureter is a rare cause of congenital hydronephrosis. The surgical correction of RCU should be performed in all patients with obstruction and hydronephrosis symptoms, lumbar pain, urinary tract infections, hematuria, or urolithiasis. Traditionally, an open surgical approach was used for the treatment of RCU. Nowadays, surgical correction of these anomalies is performed using minimally invasive techniques. We report on two cases treated with our standardized laparoscopic technique using only three 5-mm trocars. The proposed approach could be considered as the first-line treatment for RCU.

\section{New Insights and the Importance for the Pediatric Surgeon}

We present our laparoscopic technique for retrocaval ureter with a very minimally invasive procedure, demonstrating that it is an effective and safe treatment.

\section{Introduction}

Retrocaval ureter (RCU) or circumcaval ureter is a rare cause of congenital hydronephrosis. Although its exact incidence remains unknown, its estimated incidence is 1 in 1,100 live births per year, ${ }^{1}$ with a male-to-female ratio of $3: 1 .^{2}$ RCU occurs due to an unusual persistence of the right subcardinal vein positioned ventrally to the ureter and developed in the definitive inferior vena cava (IVC). However, it is sometimes associated with other IVC abnormalities. ${ }^{3}$ The lumbar ureter compression can indicate several degrees of obstruction without specific clinical presentation; therefore, many patients may be completely asymptomatic. Its clinical manifestation is usually recognized from the second to the fifth decade of life, with flank pain and recurrent urinary tract infections as the most common symptoms and hematuria and urolithiasis as

received

May 27, 2019

accepted after revision

January 10,2020

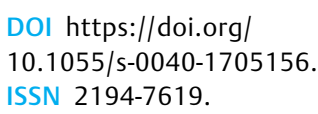

DOI https://doi.org/

10.1055/s-0040-1705156. ISSN 2194-7619.

the less common symptoms. RCU may also result in long-term renal function impairment. ${ }^{4}$ Ultrasonography, multislice computed tomography, contrast-enhanced magnetic resonance imaging (MRI), and mercapto-acetyl-triglycine (MAG3) scintigraphy are the main diagnostic tools. For many years, the gold standard treatment for RCU has been ureteral transposition with uretero-ureteric anastomosis by open surgery. However, in the last two decades, laparoscopic ureteroureterostomy has been proposed as a feasible option. ${ }^{1,5}$ Here, we performed a minimally invasive laparoscopic approach in two patients by adopting a standardized technique and demonstrated its safety and efficacy.

\section{Case Report}

From January 2016 to January 2018, two female patients with symptomatic RCU were examined. Their ages at the time of
License terms

Stuttgart · New York

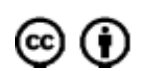




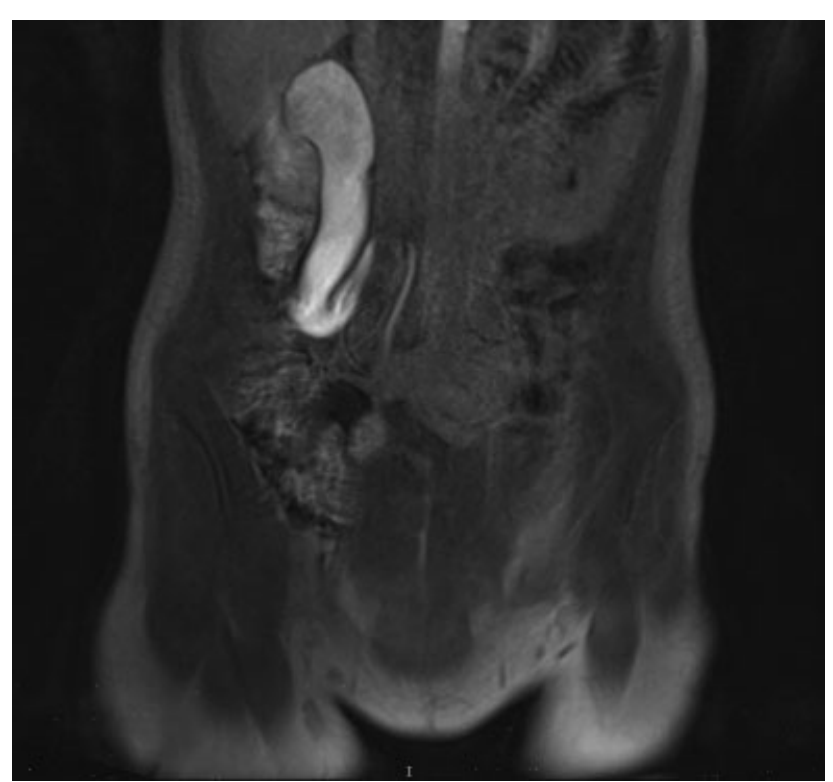

Fig. 1 Coronal view of the magnetic resonance image of the typical type 1 retrocaval ureter variant with S-shaped deformity of right lumbar ureter with hydronephrosis and dilatation of the associated proximal ureter (case 2).

surgery were 22 and 18 years. Body weights were respectively 72 and $56 \mathrm{~kg}$. Patient 1 underwent augmentation enterocystoplasty with bilateral ureteral reimplantation for spinal dysraphism at the age of 5 years. The RCU was not recognized at that time, because the refluxing ureter was grossly dilated and tortuous. Recently, she presented recurrent episodes of right flank pain and two episodes of acute pyelonephritis, which were treated with antibiotics. No reflux was present in the cystogram. Patient 2 showed intermittent gross hematuria with several episodes of flank pain since the age of 12 years. Abdominal MRI with three-dimensional reconstruction revealed that both the patients had a typical S-shaped deformity of the right lumbar ureter, according with type 1 variant of $\mathrm{RCU}^{6}$ ( - Fig. 1). The renogram for MAG3 renal scan revealed that the affected kidneys of both the patients had good renal function (split renal function of 47 and $45 \%$, respectively) with partially obstructed urine drainage. Patients underwent minimally invasive laparoscopic ureterocaval transposition with dismembered ureteroureterostomy through a transperitoneal approach using three $5-\mathrm{mm}$ trocars. Patients were positioned in 45 degrees left flank position. The pneumoperitoneum was established at $12 \mathrm{~mm} \mathrm{Hg}$. The dilated pelvis and right ureter were identified after mobilizing the right colon and opening the Gerota fascia ( - Fig. 2A). The proximal ureter was lifted on a vessel loop, and the distal ureter segment was poorly mobilized in the inter-aorto-caval region ( - Fig. 2B). The ureter was transected proximally to the IVC, and then the stenotic tract was excised ( - Fig. $2 \mathrm{C}$ ). The uretero-ureteric anastomosis was created using two running sutures ( $5 / 0$ polyglactin) after adequately spatulating the distal segment. A 4.7-Fr double-J stent was inserted laparoscopically with an antegrade technique through a percutaneous $18-\mathrm{G}$ needle (-Fig. 2D). A 10-Fr continuous suction drain was placed postoperatively. All procedures were laparoscopically completed within 195 and 172 minutes in patients 1 and 2, respectively, without perioperative complications. In addition, intracorporeal suturing took 79 and 73 minutes, and blood loss was 20 and $30 \mathrm{~mL}$ in patients 1 and 2 , respectively. The postoperative period was uneventful for both patients. The suction drain was removed after 48 and 60 hours from patients 1 and 2, respectively. Subsequently, patients 1 and 2 were discharged on the fourth and fifth postoperative day, respectively. The double-J stent was removed after 4 weeks. Renal ultrasonography and scintigraphy were performed 1 month after the double-J stent removal. Renal ultrasonography showed a resolved
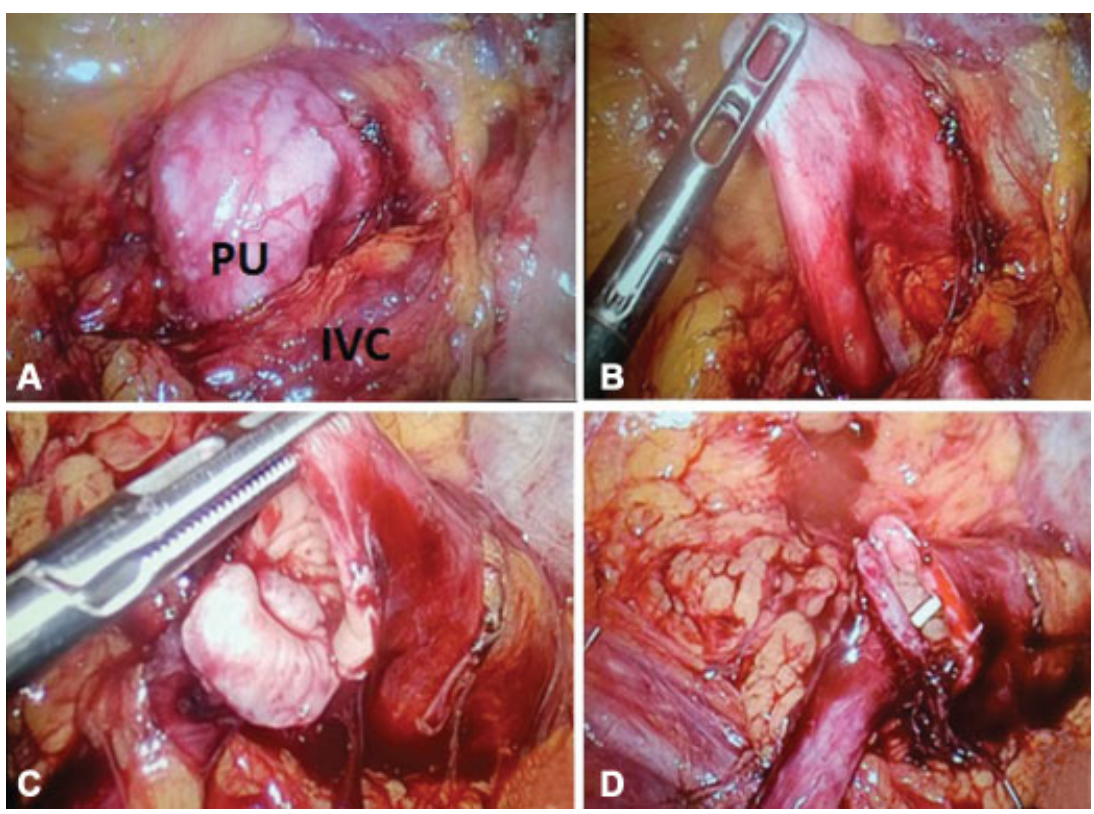

Fig. 2 Intraoperative images of the proximal dilated right ureter (case 2). (A) Dilated proximal ureter (PU) crossing the inferior vena cava (IVC). (B) Mobilization of the PU with narrowed segment under the IVC. (C) Stenotic tract of the ureter excised. (D) Uretero-ureteric anastomosis after spatulation. 
pyelocalyceal dilatation at 3-, 6-, and 12-month follow-up. Renal function was stable, and MAG3 renal scan revealed significant increase in urine drainage in both the patients at the 18-month follow-up. Patients remained symptom-free 24 months postoperatively.

\section{Discussion}

$\mathrm{RCU}$ is a rare congenital anomaly generally associated with upper urinary tract obstruction and hydronephrosis. Its typical radiological presentation is an S- or sickle-shaped appearance, due to the passage of the ureter at the posterior side of the IVC. ${ }^{5}$ According to the radiologic appearance, RCU is classified into two types. ${ }^{6}$ In type 1 (low loop, S-shaped), the dilated upper ureteral tract descends from the renal pelvis and then curves upward and medially forming a reversed J appearance in the intravenous pyelogram. The retrograde pyelogram shows a typical S-shaped outline (-Fig. 1). The level of ureteral retrocaval segment is most frequently found at the third lumbar vertebrae. In type 2 variant (high loop, sickle-shaped), the renal pelvis and upper ureter lie almost horizontal; therefore, the ureteral retrocaval segment is on the same level. Type 1 variant is more common and usually causes moderate to severe hydronephrosis, whereas type 2 is rarer with less severe hydronephrosis and does not generally cause obstruction. ${ }^{7,8}$ Both our cases were recognized as type 1 variant of RCU. The surgical correction of RCU should be performed in all patients with obstruction and hydronephrosis symptoms, lumbar pain, urinary tract infections, hematuria, or urolithiasis. Asymptomatic patients with functionally unobstructed RCUs who may even show hydronephrosis on radiological imaging do not necessarily need immediate surgical treatment. ${ }^{9}$ Our two patients presented symptoms of obstruction and hydronephrosis with urinary tract infections. Traditionally, an open surgical approach was used for the treatment of RCU. However, there was wide incision, quite long convalescence period, and delay in return to daily activities. Therefore, surgical correction of these anomalies has been attempted using minimally invasive techniques. In 1994, Baba et al described the first laparoscopic transperitoneal procedure for treatment of RCU. ${ }^{10}$ Nowadays, RCU can be approached laparoscopically by performing either trans- or retroperitoneal procedures. A few studies have reported the utility of the laparoscopic transperitoneal approach for the treatment of RCU with consistent positive results. ${ }^{11-15}$ According to Ramalingam and Selvarajan, ${ }^{11}$ transperitoneal approach is a less time-consuming, relatively easier procedure than the retroperitoneal approach because of larger operation field and very good exposure.

Our technique of RCU management utilizes only three 5-mm trocars, without any preoperative cystoscopy or indwelling ureteral catheter insertion. No critical difficulty was found in the intra-abdominal adhesions in patient 1 . The simplicity and reproducibility of our procedure are its main advantages. We believe that this is a safe, feasible, effective, and minimally invasive approach, and therefore could be considered as the first-line treatment for RCU.

\section{Conflict of Interest}

None.

\section{References}

1 Simforoosh N, Nouri-Mahdavi K, Tabibi A. Laparoscopic pyelopyelostomy for retrocaval ureter without excision of the retrocaval segment: first report of 6 cases. J Urol 2006;175(06): 2166-2169, discussion 2169

2 Soundappan SVS, Barker AP. Retrocaval ureter in children: a report of two cases. Pediatr Surg Int 2004;20(02):158-160

3 Ishitoya S, Arai Y, Waki K, Okubo K, Suzuki Y. Left retrocaval ureter associated with the Goldenhar syndrome (branchial arch syndrome). J Urol 1997;158(02):572-573

4 Hensle TW, Shabsigh A. Pyeloplasty (Anderson-Hynes). BJU Int 2004;93(07):1123-1134

5 Sun J-S, Zhang G, Lin T. Retrocaval ureter in children: a report of eight cases. West Indian Med J 2015;64(04):397-399

6 Acharya SK, Jindal B, Yadav DK, Singha S, Bagga D. Retrocaval ureter: a rare cause of hydronephrosis in children. J Pediatr Surg 2009;44(04):846-848

7 Dogan HS, Oktay B, Vuruskan H, Yavascaoglu I. Treatment of retrocaval ureter by pure laparoscopic pyelopyelostomy: experience on 4 patients. Urology 2010;75(06):1343-1347

8 Li H-Z, Ma X, Qi L, Shi T-P, Wang B-J, Zhang X. Retroperitoneal laparoscopic ureteroureterostomy for retrocaval ureter: report of 10 cases and literature review. Urology 2010;76(04):873-876

9 Liu E, Sun X, Guo H, et al. Retroperitoneoscopic ureteroplasty for retrocaval ureter: report of nine cases and literature review. Scand J Urol 2016;50(04):319-322

10 Baba S, Oya M, Miyahara M, Deguchi N, Tazaki H. Laparoscopic surgical correction of circumcaval ureter. Urology 1994;44(01): 122-126

11 Ramalingam M, Selvarajan K. Laparoscopic transperitoneal repair of retrocaval ureter: report of two cases. J Endourol 2003;17(02): 85-87

12 Tamhankar AS, Savalia AJ, Sawant AS, Pawar PW, Kasat GV, Patil SR. Transperitoneal laparoscopic repair of retrocaval ureter: our experience and review of literature. Urol Ann 2017;9(04): 324-329

13 El Harrech Y, Ghoundale O, Kasmaoui EH, Touiti D. Transperitoneal laparoscopic pyelopyelostomy for retrocaval ureter without excision of the retrocaval segment: experience on three cases. Adv Urol 2016;2016:5709134

14 Ding GQ, Xu LW, Li XD, et al. Pure transperitoneal laparoscopic correction of retrocaval ureter. Chin Med J (Engl) 2012;125(13): 2382-2385

15 Seo IY, Oh TH, Jeon SH. Transperitoneal laparoscopic ureteroureterostomy with excision of the compressed ureter for retrocaval ureter and review of literature. Investig Clin Urol 2019;60(02): 108-113 